\title{
GAS TURBINE RELIABILITY MODEL BASED ON TANGENT HYPERBOLIC RELIABILITY FUNCTION
}

\author{
Ahmed Zohair Djeddi, Ahmed Hafaifa \\ University of Djelfa, Faculty of Science and Technology, Djelfa, Algeria \\ e-mail: a_z_djeddi@univ-djelfa.dz; hafaifa.ahmed.dz@ieee.org \\ Abudura SAlam \\ University of Médéa, Faculty of Science and Technology, Médéa, Algeria \\ e-mail: abudura.salam@univ-medea.dz
}

\begin{abstract}
The present work deals with the exploration of a new model proposed for the reliability analysis of industrial production systems. This proposed model is mainly based on the tangent hyperbolic function, where the survival function is determined and used in the lifetime distribution modeling taking into account of estimation the parameters of the proposed function. On the other side, tests validation is performed using the real data of a gas turbine installation. The obtained results allow the modeling of damage effects, hence the prediction of the performance of the examined gas turbine using the proposed model gives good results in terms of validity.
\end{abstract}

Keywords: reliability estimation, reliability algorithms, lifetime distribution, Weibull distribution, availability

\section{Introduction}

Actually, the complexity of industrial plants and their equipment behavior led practically to very complex maintenance strategies that are containing a number of different tasks. Moreover, the random nature of degradation and failure that may occur makes the determination of the required strategy to fulfill the best decisions regarding their maintenance a very difficult practical task (Costa et al., 2014; Lai, 1994; Guemana et al., 2011; Rao et al., 2005; Hasumi et al., 2009). The main aim of the proposed model is to analyze and measure industrial system reliability parameters to achieve the best time determination for making decision on the maintenance actions. Whereas, in the present work, this study is based on a model of a gas turbine which is considered as the main important equipment of the compressor stations, gas pumps, oil pumps and petrol production. The reliability model proposed in this paper is based essentially on the elementary function of tangent hyperbolic which is proved in this paper to be theoretically and practically similar to the well know Weibull distribution which was introduced by the Swedish mathematician in 1951 (Weibull, 1951). The Weibull distribution is considered the first distribution used in reliability analysis due to its very big capacity to adapt a very large number of data sets. However, many lifetime distributions that have a bathtub-shaped hazard rate function have been introduced after Weibull distribution, in which the main aim was to improve the systems reliability. A survey presenting the state-of-the-art on the class of such distributions was presented by Moeini et al. (2013), Scott (1979), Lai et al. (1998, 2001, 2003).

This work proposes an exploration of a new model which will contribute to the enhancement of the industrial systems reliability. It is obvious that the hyperbolic tangent function has a curve similar to the cumulative function of the probability distribution. Hence, a new model based on a tangent hyperbolic function defined on $\Re^{+}$can be proposed. It is very clear that 
its form is similar to the form of Weibull's model. In this model, the survival function will be studied based on it.

For validation of the proposed model, a practical application has been made with real data. These data have been collected from a gas turbine operating in a natural gas transportation system which is used in the south of Algeria. The reliability approach developed in this paper allows the modeling of the effects of damage to predict the performance of the examined gas turbine operation and give good results in terms of validity compared with the Weibull approach.

\section{Reliability model based on the tangent hyperbolic function}

The availability of the industrial equipment control allows the industries to act positively on the conformity of production, the exploitation costs and the production successful competitiveness. Indeed, the life managing of the equipment that is being used in oil and gas facilities is based on taking into account the aspects of aging, the economic and regulatory factors in order to optimize the operation, maintenance and lifespan of the system structures and components. The main goal is to maintain the level of safety and reliability as well as to maximize the investment return on the overall lifespan. The reliability of industrial systems is always essential and attracts much attention among scientific researches. It is especially important for components whose failure can cause major problems in terms of maintainability, availability and security (Halimi et al., 2014; Hafaifa et al., 2013a,b; Sturges, 1926; Ruji, 1990; Trofimov et al., 1978). As it is aforementioned, a new model based on the tangent hyperbolic function is proposed to be used for the analysis and the study of the survival function in the reliability modeling. The tangent hyperbolic function considered in this paper is defined as a function on $\Re^{+}$(Fig. 1). It is expressed as follows

$$
F(x)= \begin{cases}0 & \text { for } \quad x \in \Re^{+} \\ \tanh (x) & \text { for } \quad x \geqslant 0\end{cases}
$$

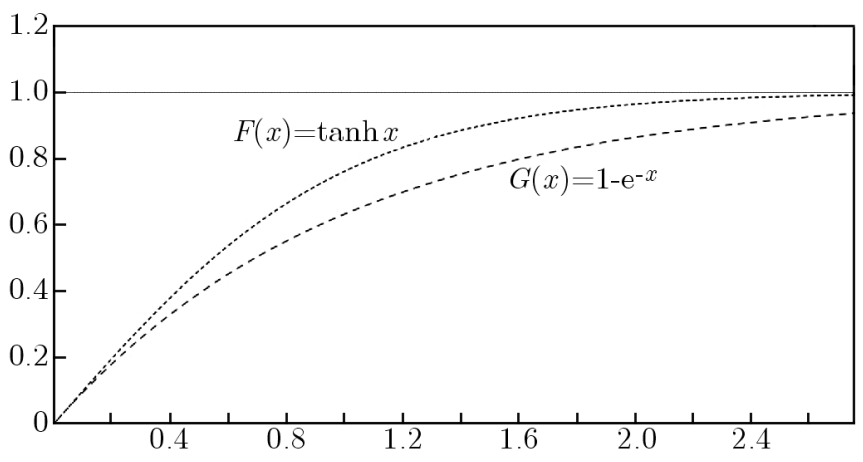

Fig. 1. Tangent hyperbolic function

To assess the impact of the aging effects on the equipment by annual frequency and to identify the sensitive equipment and the requested strategies for service priority to manage the risks that are associated with the age of the facilities, a cumulative distribution function is used. In this case it is the limit of $F(x)$ equal to 1 when $x$ tends to +infinity and is equal to 0 when $x$ tends to 0 . The proposed function is a non-decreasing and a right-continuous function, so the proposed tangent hyperbolic function is fulfils these requirements and it presents a good choice as a candidate cumulative distribution function (Costa et al., 2014; Guaily and Epstein, 2013)

$$
\lim _{x \rightarrow 0} F(x)=0 \quad \lim _{x \rightarrow+\infty} F(x)=1
$$


The distribution lifespan in the proposed tangent hyperbolic distribution form is deduced from the Weibull distribution. The survival function is represented as follows

$$
R(t)=1-\tanh (\lambda t)^{\beta}
$$

where $\beta$ is the shape parameter and $\lambda$ is the scale parameter. The cumulative distribution function is expressed as follows

$$
F(t)=1-R(t)=\tanh (\lambda t)^{\beta}
$$

Based on the derivative of the last function, the probability density function is obtained as follows:

$$
f(t)=\frac{d F(t)}{d t}=\frac{(\lambda t)^{\beta-1} \lambda \beta}{\cosh ^{2}(\lambda t)^{\beta}}
$$

The hazard function is determined in the flowing equations

$$
P_{r}(t<T \leqslant t+\Delta t \mid T>t)=\frac{f(t)}{R(t)} \Delta t=h(t) \Delta t
$$

where

$$
h(t)=\frac{f(t)}{R(t)}=\frac{(\lambda t)^{\beta-1} \frac{\lambda \beta}{\cosh ^{2}(\lambda t)^{\beta}}}{1-\tanh (\lambda t)^{\beta}}
$$

Using a variable converting the reliability $R(t)$ replaced by the hyperbolic function, $h(t)$ can be rewritten using exponential form as follows

$$
h(t)=\frac{2 \lambda \beta(\lambda t)^{\beta-1}}{1+\mathrm{e}^{-2(\lambda t)^{\beta}}}
$$

\subsection{Hazard and density functions}

The hazard function in the proposed tangent hyperbolic model is expressed by equation (2.7) and is shown in Fig. 2. It is obvious that the curve of this function depends only on the parameter $\beta$. Therefore, the curve of this function can be discussed based on the value of $\beta$ :

- In the case when $\beta=1$, the hazard function tends quickly to $2 \lambda$

$$
\lim _{t \rightarrow \infty} h(t)=2 \lambda
$$

- In the case when $\beta=2$, the hazard function becomes

$$
h(t)=\frac{4 t \lambda^{2}}{1+\mathrm{e}^{-2(\lambda t)^{2}}} \rightarrow 4 t \lambda^{2}
$$

The hazard function $h(t)$ is a straight line with slope equal to $4 \lambda^{2}$.

- In the case when $0<\beta<1$, the hazard function becomes

$$
h(t) \rightarrow 2 \lambda \beta(\lambda t)^{\beta-1}
$$

The hazard function curve follows $t^{p}$ form with $-1<p<0$.

- In the case when $\beta>2$, the hazard function curve follows $t^{p}$ with $p>1$ (convex form).

- In the case when $1<\beta<2$, the hazard function curve follows $t^{p}$ with $0>p>1$ (concave form).

It can be concluded that the curves of the hazard function have their Weibull equivalents curves. On the other side, the density function of the proposed tangent hyperbolic model presented in equation (2.5) is presented in Fig. 3 for different values of $\beta$. 


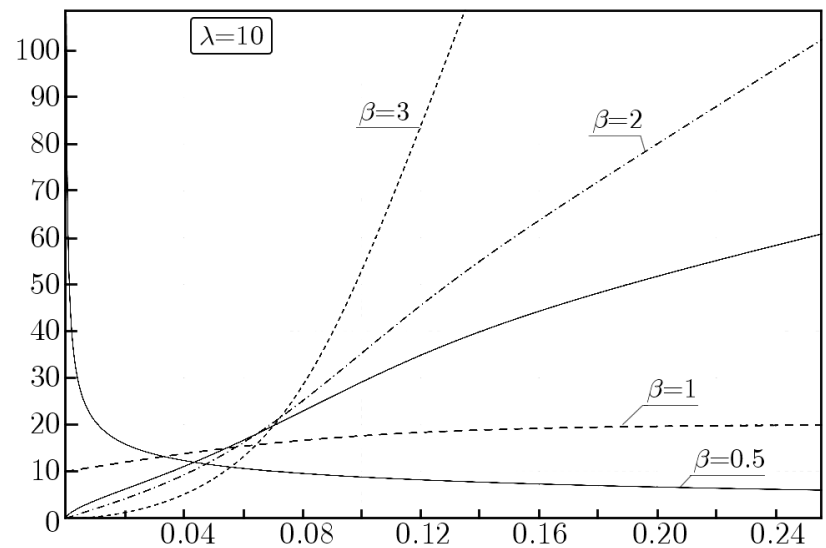

Fig. 2. Hazard function determined using the tangent hyperbolic algorithm

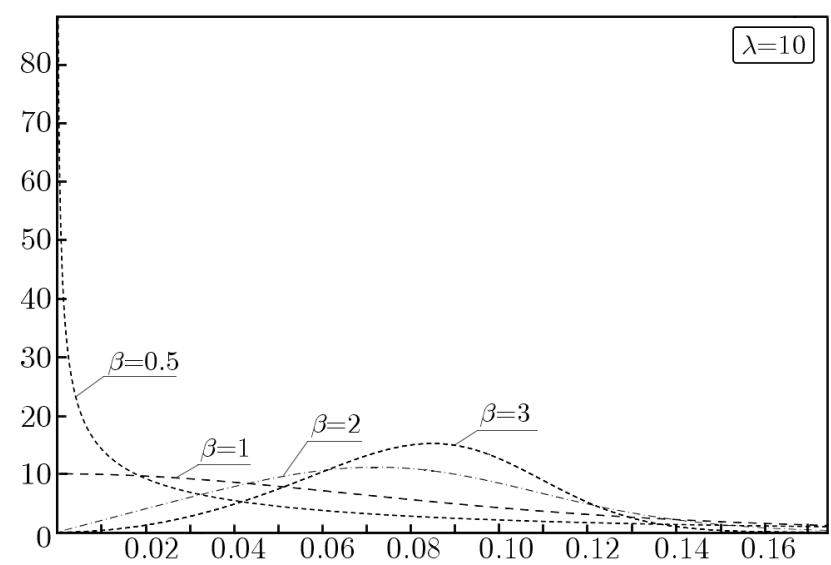

Fig. 3. Density function found using the hyperbolic model

\section{Application results}

The data used in this paper are taken from the data history of a gas turbine (PGT10) used for natural gas transportation and installed in a gas plant located in the south of Algeria. Those data are presented in Table 1.

Table 1. Examined gas turbine data

\begin{tabular}{|c|c|c|c|c|c|c|c|c|c|c|}
\hline$N$ & 1 & 2 & 3 & 4 & 5 & 6 & 7 & 8 & 9 & 10 \\
\hline TBF $^{*}$ & 240 & 264 & 384 & 552 & 624 & 648 & 696 & 720 & 720 & 768 \\
\hline$N$ & 11 & 12 & 13 & 14 & 15 & 16 & 17 & 18 & 19 & 20 \\
\hline TBF & 768 & 792 & 960 & 1272 & 1344 & 1464 & 1632 & 1680 & 1776 & 1944 \\
\hline$N$ & 21 & 22 & 23 & 24 & 25 & 26 & 27 & 28 & 29 & 30 \\
\hline TBF & 1968 & 1992 & 2064 & 2136 & 2208 & 2376 & 2448 & 2448 & 2472 & 2664 \\
\hline$N$ & 31 & 32 & 33 & 34 & 35 & 36 & 37 & 38 & 39 & 40 \\
\hline TBF & 2832 & 2880 & 3000 & 3600 & 3672 & 3720 & 4272 & 4656 & 5592 & 5856 \\
\hline
\end{tabular}

* TBF is the Time Between Failures given in hours

\subsection{Esytimation of the model parameters}

Using equation (2.4), the inverse of the tangent hyperbolic function can be obtained as follows

$\tanh ^{-1} y=(\lambda t)^{\beta}$ 
The tangent hyperbolic can be eliminated from this equation based on the following well known expression

$$
\tanh ^{-1} y=\frac{1}{2} \log \frac{1+y}{1-y}
$$

Furthermore, if the log is used to the two members of equation (3.1), the obtained expression is as follows

$$
\log \left(\frac{1}{2} \log \frac{1+y}{1-y}\right)=\beta \log \lambda+\beta \log t
$$

which can be transformed into a linear expression

$$
Y=A+B X
$$

where

$$
Y=\log \left(\frac{1}{2} \log \frac{1+y}{1-y}\right) \quad X=\log t \quad A=\beta \log \lambda \quad B=\beta
$$

To estimate $A$ and $B$, any graphical or analytical methods can be used, a possible approach is the simple regression analysis using (3.2). The estimation of the parameters based on the least-squares fit is shown in Fig. 4. The parameters of the examined gas turbine using the proposed approach are presented in Table 2. The obtained estimated parameters are $\lambda=0.00034$ and $\beta=1.24568$.

Table 2. The obtained parameters for the examined gas turbine

\begin{tabular}{|c|c|c|c|c|}
\hline & Estimate & Std. Error & $t$ value & $\operatorname{Pr}(>|t|)$ \\
\hline \hline$\beta$ & 1.24569 & 0.03435 & 36.27 & $<2 \mathrm{E}-16$ \\
\hline$\beta \log \lambda$ & -7.98420 & 0.02801 & -285.07 & $<2 \mathrm{E}-16$ \\
\hline
\end{tabular}

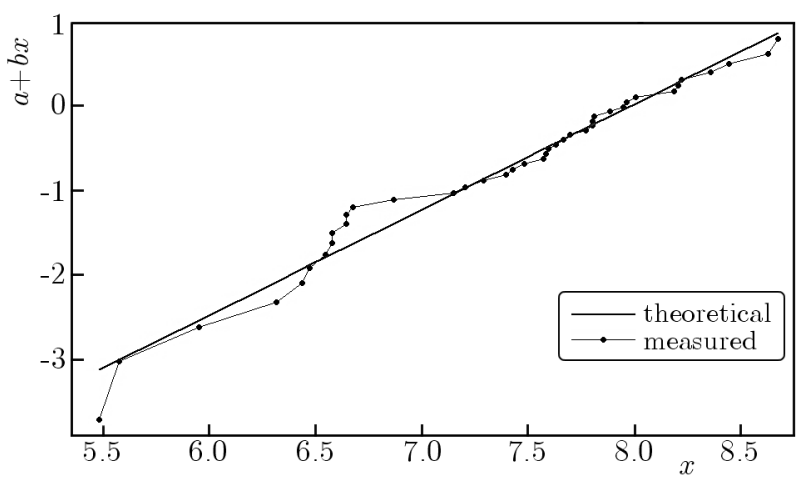

Fig. 4. The distribution function of the examined gas turbine determined using the hyperbolic algorithm

The plots of $Y$ versus $X$ which are related to the cumulative distribution function and time for the examined gas turbine determined using the proposed tangent hyperbolic algorithm are shown in Fig. 4, where the plotted data are scattered close to the fitted straight line. It can be said that this model is acceptable (Murthy et al., 2004; Yang and Scott, 2013).

\subsubsection{Cumulative distribution function}

The cumulative distribution function of the examined gas turbine found using the tangent hyperbolic model is shown in Fig. 5. The plotted data are scattered close to the fitted curve. Following the cumulative distribution function expressed in equation (2.4), the parameters $\lambda=0.00034$ and $\beta=1.24568$. 


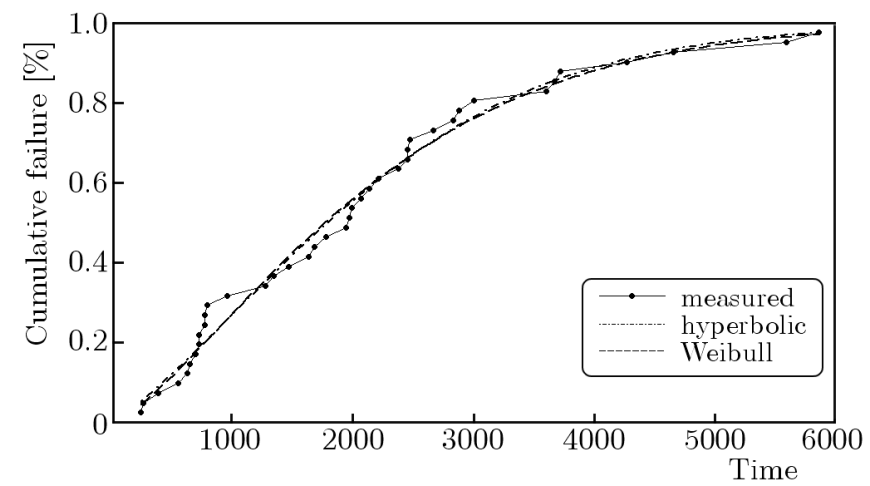

Fig. 5. The cumulative failure rate of the examined gas turbine using the hyperbolic model

\subsubsection{Density and hazard functions}

The density function and the hazard function of the examined gas turbine determined by using the tangent hyperbolic function model are shown respectively in Figs. 6 and 7. These functions are obtained by using equation (2.5) and taking into account the estimated parameter values $\lambda=0.00034$ and $\beta=1.24568$.

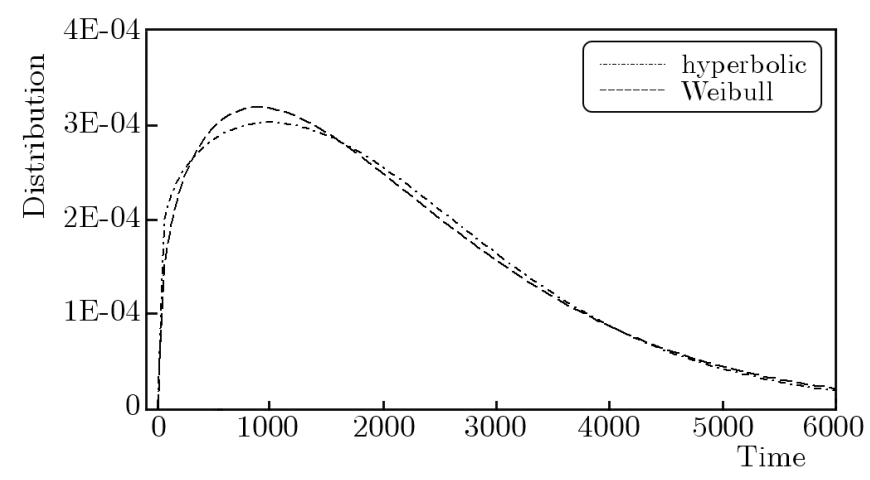

Fig. 6. The distribution function of the model of the examined gas turbine using the hyperbolic model

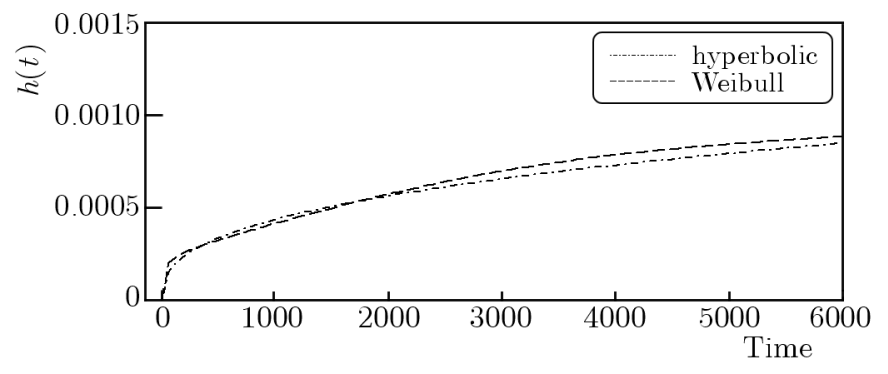

Fig. 7. The hazard function of the examined gas turbine using the hyperbolic model

\subsubsection{Mean time between failures}

The Mean Time Between Failures (MTBF) is the predicted elapsed time between inherent failures of the system during exploitation (Yang and Scott, 2013) which is expressed as follows

$$
\operatorname{MTBF}=\int_{0}^{+\infty} t f(t) d t=\int_{0}^{+\infty} t \frac{(\lambda t)^{\beta-1} \lambda \beta}{\cosh ^{2}(\lambda t)^{\beta}} d t
$$


It is worth noting that there is an alternative way for computing the expected value (Murthy et al., 2004)

$$
\operatorname{MTBF}=\int_{0}^{+\infty} S(t) d t=\int_{0}^{+\infty} 1-\tanh (\lambda t)^{\beta} d t
$$

Table 3

\begin{tabular}{|c|c|c|}
\hline Model & Weibull & Hyperbolic function \\
\hline MTBF & $2088.623 \mathrm{~h}$ & $2075.40 \mathrm{~h}$ \\
\hline
\end{tabular}

\section{Conclusion}

The goal achieved in this paper is concerned with industrial reliability, which requires the specialists to be more competitive and more responsive to variable market conditions. It is obvious that to achieve better management of the facilities performance, it is necessary to improve the reliability. It has been shown that with the application of the proposed method based on the tangent hyperbolic function good results can be obtained. The main objective is to analyze and to measure the reliability parameters of the examined system in order to determine the best time to take maintenance action based on reliability analysis. The developed in this paper reliability approach allows the modeling of the damage effects to predict the performance of the examined gas turbine operation and to lead to good results in terms of validity compared with the Weibull approach. This model can be improved to have a bathtub-shaped hazard form.

\section{Refernces}

1. Costa F.M.P., Rocha A.M.A.C., Fernandes E.M.G.P., 2014, An artificial fish swarm algorithm based hyperbolic augmented Lagrangian method, Journal of Computational and Applied Mathematics, 259, Part B, 868-876

2. Guaily A.G., Epstein M., 2013, Boundary conditions for hyperbolic systems of partial differentials equations, Journal of Advanced Research, 4, 4, 321-329

3. Guemana M., Aissani S., Hafaifa A., 2011, Use a new calibration method for gas pipelines: an advanced method improves calibrating orifice flowmeters while reducing maintenance costs, Hydrocarbon Processing Journal, 90, 8, 63-68

4. Hafaifa A., Belhadef R., Guemana M., 2013a, Reliability model exploitation in industrial system maintainability using expert system evaluation, Proceedings of the 4 th International Conference on Integrity, Reliability and Failure, IRF2013, Funchal, Madeira, Portugal, 387-388

5. Hafaifa A., Guemana M., Daoudi A., 2013b, Vibrations supervision in gas turbine based on parity space approach to increasing efficiency, Journal of Vibration and Control, doi: $10.1177 / 1077546313499927$

6. Halimi D., Hafaifa A., Bouali E., 2014, Maintenance actions planning in industrial centrifugal compressor based on failure analysis, Journal of Maintenance and Reliability, 16, 1, 17-21

7. Hasumi T., Aкimoto T., Aizawa Y., 2009, The Weibull-log Weibull distribution for interoccurrence times of earthquakes, Physica A: Statistical Mechanics and its Applications, 388, 4, 491-498

8. LAI C.D., 1994, Tests of univariate and bivariate stochastic ageing, IEEE Transactions on Reliability, 43, 2, 233-241 
9. LAi C.D., Moore T., XIE M., 1998, The beta integrated model, Prococeding of the International Workshop on Reliability Modeling and Analysis: from Theory to Practice, 153-159

10. Lai C.D., Xie M., Murthy D.N.P., 2001, Bathtub shaped failure rate distributions, Handbook in Reliability, 20, 69-104

11. Lai C.D., Xie M., Murthy D.N.P., 2003, A modified Weibull distribution, IEEE Transactions on Reliability, 52, 1, 33-37

12. Moeini A., Jenab K., Mohammadi M., Foumani M., 2013, Fitting the three-parameter Weibull distribution with Cross Entropy, Applied Mathematical Modelling, 37, 9, 6354-6363

13. Murthy P.D.N., Xie M., Jiang R., 2004, Weibull Models, John Wiley \& Sons

14. Rao C.R., Wegman E.J., Solka J.L., 2005, Handbook of Statistics: Data Mining and Data Visualization, Volume 24 de Handbook of Statistics, Elsevier Science

15. Ruji H., 1990, State space tree method and exact decomposition algorithm for finding network overall reliability, Journal of Electronics (China), 7, 4, 296-305

16. Scott D.W., 1979, On optimal and data-based histograms, Biometrika, 66, 3, 605-610

17. Sturges H.A., 1926, The choice of a class interval, Journal of the American Statistical Association, 21, 153, 65-66

18. Trofimov N.G., Kravchenko B.A., Kramarovskit B.I., Baturov V.B., Kostina G.N., 1978, Increasing the strength and reliability of turbine blades by thermoplastic hardening methods, Strength of Materials, 10, 8, 990-996

19. Weibull W., 1951, A statistical distribution function of wide applicability, Journal of Applied Mechanics, 1, 18, 293-297

20. Yang J., Scott D.W., 2013, Robust fitting of a Weibull model with optional censoring, Computational Statistics and Data Analysis, 67, 1, 149-161

Manuscript received October 3, 2014; accepted for print March 11, 2015 